\title{
Percepciones sobre programas de formación e institucionalidad en Seguridad Alimentaria y Nutricional (SAN) en Honduras
}

\author{
Carlos Roberto Ardón ${ }^{1}$ y Ana Afonso Gallegos ${ }^{2}$
}

\begin{abstract}
Resumen. El hambre es un problema que afecta en la actualidad a más de 1,000 millones de personas en el mundo, de los cuales 53 millones están en América Latina. Honduras no es ajena a ese problema, el 12\% de su población no dispone de los alimentos suficientes para una vida saludable. En los últimos años, organizaciones de prestigio internacional han estudiado el problema y planteado diversas estrategias de solución; es vital el compromiso político de los estados y la participación de instituciones y personas con voluntad y capacidad de aplicar dichas estrategias en los territorios más desfavorecidos. Con el objetivo de conocer la situación en Honduras sobre programas de formación e institucionalidad en Seguridad Alimentaria Nutricional (SAN), se realizó un estudio de percepciones entre profesionales de distintos sectores y con vinculación comprobada con el tema. La educación superior en el país es considerada entre poco y nada pertinente con la SAN por la mayoría de los encuestados. Un alto porcentaje de ellos cree que se debe aumentar la oferta de formación en SAN y definen el nivel de pregrado como más importante. Existen diversas instancias de coordinación alrededor del tema y la mayoría visualiza diversos beneficios de las mismas, pero se necesita más coordinación para incidir a nivel de políticas públicas. La participación de las universidades en estos espacios es considerada poco relevante; sugiriendo que deberían jugar un rol más determinante, especialmente en la investigación y generación de información. Por la gravedad del problema y con el objetivo de una mayor sensibilización e integralidad de esfuerzos, además de la creación de programas específicos, se sugiere que la SAN sea abordada de manera transversal en el sistema educativo nacional.
\end{abstract}

Palabras clave: Pertinencia educativa, políticas públicas, seguridad alimentaria.

Abstract. Hunger is a problem that currently affects more than one billion people worldwide, which 53 million are in Latin America. Honduras isn't stranger to this problem, $12 \%$ of its population doesn't have enough food for healthy life. In the latest years, prestigious international organizations have studied the problem and proposed different solution strategies. It's very important the political commitment of governments and the participation of institutions and people with the ability to apply these strategies in the most disadvantaged territories. In order to know the Honduras situation about the training programs and institutions working in food security, it was made a perceptions study among Honduran professionals from different sectors and with extensive experience in the subject. Higher Education is considered between little and not pertinent to the food security by the plenty of request. A high percent of them believes it's necessary to increase the offer training in food security and they define university degree as the most important level. There are several institutions working around food security and the majority visualizes various benefits of it, however, more work is needs to influence public policies. The involvement of universities in these institutions is considered slightly relevant; suggesting that universities take part in research processes and knowledge transfer in relation to the subject. Because the intensity of the problem and to achieve greater awareness, moreover the training specific programs, it's appropriate that food security can be studied in whole educational national system.

Key words: Educational pertinence, food security, public policies.

1 Ing. Agrónomo, Máster en Desarrollo Rural. Alumno programa doctorado en Planificación y Gestión de Proyectos de Desarrollo Rural Sostenible. Universidad Politécnica de Madrid. España. Email: cardon682003@yahoo.com

2 Ing. Agrónomo, Doctora. Docente e investigadora del grupo GESPLAN de la Universidad Politécnica de Madrid. España, Email: ana.afonso@fgupm.es . 


\section{Introducción}

En la Cumbre Mundial sobre la Alimentación (CMA) en Roma en 1996 se definió seguridad alimentaria como la situación en donde las personas tienen en todo momento acceso físico y económico a suficientes alimentos inocuos y nutritivos para satisfacer sus necesidades nutricionales; también se destacó que en ese momento, 800 millones de personas en todo el mundo no disponían de esos alimentos y se planteó el compromiso de reducir a la mitad ese número para el 2015 (FAO 1996). Muchos esfuerzos se han realizado desde entonces, pero los resultados no son los esperados. Actualmente existen 1,020 millones de personas en todo el mundo padeciendo hambre (FAO 2009a). En América Latina y El Caribe se estima que hay 53 millones de personas (10\% de la población total) en esa penosa situación (FAO 2009b).

En Honduras, los indicadores muestran una situación preocupante, de acuerdo a datos de CEPAL (2009), en 2007 el porcentaje de población viviendo en pobreza era de $68.9 \%$ con una mayor incidencia en las zonas rurales, donde $78.8 \%$ de la población vive en esa condición. Otro elemento muy importante es la desnutrición de la población, la misma CEPAL estima que en 2008 el porcentaje de niños menores de cinco años en estado de malnutrición en Honduras era del 25\%. Según la FAO (2008) en Honduras el porcentaje de personas subnutridas en relación al total de la población era de $12 \%$ en el período 2003-2005; una mejoría en relación al período 1995-1997 que era de 16\%. Esta situación tiene un costo elevado para el país, de acuerdo a un estudio de la Comisión Económica para América Latina y El Caribe (CEPAL) y del Programa Mundial de Alimentos (PMA), el costo total de la desnutrición en Honduras en el 2004 fue de 780 millones de dólares, lo que representa un $10.6 \%$ del Producto Interno Bruto (PIB) y un $81 \%$ del gasto social para ese año. El 93\% de ese costo, está asociado con la pérdida de productividad por mortalidad y menos años de escolaridad (CEPAL y PMA 2007). Medrano y Vallaure (2006) también analizan el costo del hambre y destacan que el estado nutricional de la población tiene un impacto directo en la economía de los países. Este impacto es por dos vías; en primer lugar el incremento en el gasto para atender las personas en estado de subnutrición (sanidad pública) y en segundo lugar la disminución de la capacidad productiva de las personas subnutridas. También tiene una relación muy fuerte el estado nutricional de las personas con el rendimiento escolar y por lo tanto en el nivel educativo del país.

Hay diversas formas de evaluar la seguridad alimentaria, Bingxin Yu et al. (2010), clasifican los países considerando cinco aspectos: consumo de alimentos, producción de alimentos, importación de alimentos, distribución de alimentos y potencial agrícola. En este análisis Honduras se ubica en el grupo de países con baja seguridad alimentaria, pero destacan que el país tiene un potencial agrícola que bien aprovechado podría contribuir de gran manera a superar el problema. De acuerdo a la clasificación de FAO (2010), Honduras es parte de los Países con Bajos Ingresos y con Déficit de Alimentos (PBIDA), un indicador que considera el ingreso per cápita y el comercio neto de alimentos. Los países en esta situación corren un gran riesgo; en primer lugar el crecimiento de las importaciones de alimentos puede afectar la producción nacional y en segundo lugar, por estar más expuestos a los mercados mundiales, un aumento en los precios de los alimentos puede provocar desabastecimientos que resulten en crisis alimentarias.

La inseguridad alimentaria es un problema complejo con una diversidad de factores políticos, sociales, económicos y ambientales que lo favorecen; estos factores han sido ampliamente estudiados y en los últimos años se han planteado diferentes estrategias de solución. La Organización de las Naciones Unidas para la Agricultura y la Alimentación (FAO), fundada en 1945 y cuyo mandato es mejorar la nutrición, aumentar la productividad agrícola, elevar el nivel de vida de la población rural y contribuir al crecimiento de la economía mundial, planteó en 2003 el enfoque de doble vía para combatir el hambre en el mundo. La primera vía plantea el fortalecimiento de la productividad y los ingresos especialmente del pequeño agricultor en los países pobres; facilitando el acceso a tecnología agrícola de bajo costo, mejora de infraestructuras rurales, sistemas de riego, desarrollo de mercados e investigación y extensión agrícola. La segunda vía consiste en proporcionar un acceso directo a los alimentos en situaciones de extrema necesidad a través de almuerzos y huertos escolares, alimentos por trabajo, comedores populares, bancos de alimentos, raciones de emergencia y ayudas al desempleo. Con la 
aplicación de estas acciones se espera influir sobre las causas y consecuencias del hambre y la pobreza, brindando soluciones en el corto, mediano y largo plazo. Es importante destacar que las dos vías han sido concebidas para llevarse a cabo de manera complementaria e integrada, de esa manera se logra suplir las necesidades alimentarias inmediatas de los sectores más vulnerables de la población y al mismo tiempo se dinamiza la agricultura en las zonas rurales, lo que resultará en un crecimiento de la economía en dichos territorios (FAO 2004).

Con el objetivo de desarrollar un plan de acción para el logro de los Objetivos del Milenio, en 2002 se conformó en las Naciones Unidas, el Millennium Project, compuesto por 10 grupos de trabajo en varios temas y donde participan organismos de Naciones Unidas, gobiernos, instituciones académicas, instituciones financieras internacionales, organizaciones no gubernamentales y el sector privado. En 2005, el grupo de trabajo contra el hambre publicó el documento "Halving Hunger: It can be done”, en el cual plantea una serie de acciones para reducir el hambre a la mitad en 2015 y eventualmente erradicarla. Dicha propuesta considera como elementos claves el compromiso político para la acción, el aumento de ingresos y productividad de agricultores en inseguridad alimentaria, la mejora de la nutrición, la reducción de la vulnerabilidad de las personas hambrientas a través de redes productivas, la creación de mercados para los más pobres y la restauración y/o conservación de los recursos naturales esenciales para la seguridad alimentaria (UN Millennium Project 2005).

La crisis alimentaria en 2008 motivó que en enero 2009 se llevara a cabo en Madrid la Reunión de Alto Nivel sobre Seguridad Alimentaria Nutricional (RANSA), donde participaron representantes de 126 países entre gobiernos, sociedad civil, sindicatos, sector privado, universidades, agencias donantes y organismos multilaterales. En dicha reunión fueron ratificadas las metas y estrategias de apoyo a la SAN aprobadas en cumbres anteriores, especialmente se reafirmó el enfoque de doble vía como medida eficaz para resolver el problema del hambre en el mundo. Además, se subrayó la importancia de la voluntad política de los estados para apoyar a las personas más desfavorecidas, la inclusión de la sociedad civil y el sector privado en los foros de discusión y el apoyo a la agricultura como elemento esencial en la lucha contra la inseguridad alimentaria. Otro acuerdo muy importante fue el inicio de un proceso de consulta para la creación de una alianza global para la agricultura, seguridad alimentaria y la nutrición (RANSA 2009).

En noviembre de 2009 se desarrolló en Roma, Italia, una nueva cumbre mundial sobre la alimentación. En este evento ratificaron las metas de las cumbres anteriores y el compromiso de erradicar el hambre en el mundo en el menor tiempo posible. También ratificaron las estrategias para el combate del problema, dentro de las cuales destaca el apoyo a la agricultura y al desarrollo rural. Estos elementos son determinantes, ya que se estima que al 2050 la producción agrícola tendrá que aumentar $70 \%$ para alimentar a toda la población mundial. Se determinó que la información es clave para la toma de decisiones acertadas y por tal motivo es urgente revitalizar los sistemas nacionales de investigación y mejorar el acceso al conocimiento; además del compromiso político y la cooperación internacional (FAO 2009c)

Lo anterior indica que organismos de gran importancia a nivel mundial, han trazado el camino a recorrer para reducir y posteriormente eliminar el hambre en el mundo. Las acciones concretas están ya definidas, pero deben existir en cada país las condiciones que faciliten su implementación; una de esas condiciones es la voluntad de los gobiernos, traducida en un marco de políticas públicas adecuadas. Es determinante también que existan personas e instituciones con la capacidad de entender y aplicar las acciones propuestas en los territorios donde el problema está presente. En tal sentido, es vital la formación de profesionales que puedan desempeñarse con éxito en instituciones del sector público y privado.

En la formación de profesionales desempeña un rol determinante la educación superior. Correa (2000), expresa que el bienestar de las comunidades rurales depende en gran medida de la formación de profesionales en universidades y centros de investigación. En la Conferencia Regional de Educación Superior en América Latina y el Caribe 2008, se concluyó que las universidades de la región deben involucrarse en la lucha contra la pobreza y el hambre; además de la prevención del cambio climático, la crisis energética y la promoción de una cultura de paz (UNESCO 2008).

Está claro entonces que: el hambre es uno de los 
grandes problemas de la sociedad actual; en Honduras los indicadores demuestran una situación complicada; hay consenso internacional sobre las estrategias de solución; se requiere de personas e instituciones capaces de aplicar esas estrategias; y la educación superior desempeña un importante papel en dicho proceso. Con esos antecedentes y con el objetivo de conocer más en detalle la situación en Honduras sobre programas de formación, institucionalidad y marco de políticas en SAN, se ha realizado un estudio de percepciones entre profesionales vinculados a la seguridad alimentaria. Además de un mejor entendimiento de la realidad nacional, la investigación también ha permitido plantear propuestas de mejora a la situación actual.

\section{Metodología}

El estudio se realizó con una encuesta a un grupo de expertos con conocimiento de la realidad hondureña que participan en organizaciones de apoyo a la SAN. Los listados con los nombres de los expertos fueron facilitados por personal del Programa Especial de Seguridad Alimentaria (PESA). Se logró encuestar a 55 personas, que representan $65 \%$ de los incluidos en los listados obtenidos.

De las personas que respondieron la encuesta, $67 \%$ de ellos son profesionales que trabajan en organizaciones no gubernamentales y otros organismos de la cooperación internacional, el 69\% se desempeñan como directores de programas y/o proyectos o como personal técnico (Cuadro 1).

Cuadro 1. Porcentaje por sector y cargo de las personas que respondieron la encuesta.

\begin{tabular}{lcccccr}
\hline \multirow{2}{*}{ Sector al que pertenecen } & \multicolumn{7}{c}{ Cargo que desempeñan } \\
\cline { 2 - 7 } & $\begin{array}{c}\text { Director de } \\
\text { organización }\end{array}$ & $\begin{array}{c}\text { Director de } \\
\text { programa/ } \\
\text { proyecto }\end{array}$ & $\begin{array}{c}\text { Personal } \\
\text { técnico }\end{array}$ & $\begin{array}{c}\text { Monitoreo y } \\
\text { evaluación }\end{array}$ & $\begin{array}{c}\text { Personal } \\
\text { docente }\end{array}$ & Total \\
\hline Sector público & 6 & 0 & 9 & 3 & 4 & 22 \\
ONG & 8 & 14 & 11 & 0 & 0 & 33 \\
Cooperación Internacional & 0 & 6 & 23 & 5 & 0 & 34 \\
Institución académica & 0 & 0 & 6 & 0 & 5 & 11 \\
Total & $\mathbf{1 4}$ & $\mathbf{2 0}$ & $\mathbf{4 9}$ & $\mathbf{8}$ & $\mathbf{9}$ & $\mathbf{1 0 0}$ \\
\hline
\end{tabular}

La encuesta contenía 25 preguntas de variado formato: evaluación, priorización y preguntas abiertas. La misma permitió recolectar información sobre los siguientes elementos:

- Pertinencia de la educación superior en Honduras en relación a la SAN.

- Cantidad y calidad de oferta de formación en SAN a nivel de pregrado y postgrado en Honduras.

- Oferta de formación en SAN a nivel de postgrado en Centroamérica.

- Propuestas de mejora ante la situación actual y priorización de diferentes niveles de formación.

- Conocimiento y valoración de la formación en competencias.

- Conocimiento de instancias de coordinación de la SAN, beneficios tangibles de su existencia y participación de universidades en dichos espacios.

- Generación de información, calidad de la misma y responsables de producirla.

- Percepción sobre marco de políticas en relación a la SAN.

Las encuestas fueron tabuladas y analizadas en el programa SPSS versión 16. Para la mayoría de las preguntas se utilizó estadística descriptiva. En las preguntas cuyo fin era asignar una calificación sobre un determinado tema, se empleó una escala cualitativa, procurando facilitar las suficientes opciones para la diversidad de criterios que normalmente se encuentran en una población. En las preguntas de priorización se solicitó a los participantes ordenar las opciones de mayor a menor importancia en una escala cualitativa, 
asignando el valor 1 a la opción de mayor importancia y así en orden ascendente hasta completar el número de posibilidades. Posteriormente, para calcular el grado de importancia, las respuestas en cada una de las opciones se multiplicaron por un valor diferente en cada caso y se sumaron los resultados obtenidos por cada opción. El resultado es una comparación entre grado de importancia (mayor a menor) percibido sobre diferentes opciones. En el cuadro 2 se muestra la escala utilizada para calcular el grado de importancia en cada caso.

Cuadro 2. Escala utilizada para valorar nivel de importancia.

\begin{tabular}{lc|lc}
\hline Preguntas con cuatro opciones & & Preguntas con cinco opciones & \\
\hline Escala cualitativa & Valor & Escala cualitativa & Valor \\
\hline 1 Extremadamente importante & 1.00 & 1 Extremadamente importante & 1.00 \\
2 Muy importante & 0.75 & 2 Muy importante & 0.80 \\
3 Bastante importante & 0.50 & 3 Bastante importante & 0.60 \\
4 Poco importante & 0.25 & 4 Medianamente importante & 0.40 \\
& & 5 Poco importante & 0.20 \\
\hline
\end{tabular}

Además de la recolección de información primaria por medio de la encuesta, también se realizó una revisión bibliográfica sobre los temas abordados. Dicha información se presenta de manera conjunta con los resultados de la consulta y su objetivo es enriquecer el estudio y profundizar sobre algunos elementos que se consideran claves dentro del mismo.

\section{Resultados y Discusión}

Los resultados se presentan en dos apartados, tal y como estaba estructurado el instrumento de consulta. El primero de ellos detalla aspectos sobre la oferta de formación en SAN y propuestas de mejora; el segundo, ilustra los resultados sobre la institucionalidad en el país, el rol de los diferentes actores y el marco de políticas existentes.

\section{Pertinencia y oferta de formación en Seguridad Alimentaria Nutricional}

Pertinencia de la educación universitaria. La pertinencia educativa se definió en la Conferencia Regional sobre Políticas y Estrategias de la Educación Superior en ALC celebrada en la Habana, Cuba en 1996 como "El rol que cumple y el lugar que ocupa la educación superior en función de las necesidades y demandas de los diversos sectores sociales" (UNESCO 1996). Izquierdo y Márquez (2000) explican la pertinencia como la situación en donde los profesionales que egresan del sistema educativo tienen la oportunidad de incorporarse al sector productivo de la sociedad. Para que la educación superior sea pertinente no sólo basta con tener programas, personal e infraestructura de calidad, es necesario que todos sus componentes estén en estrecha relación con los diferentes actores de la sociedad y que sus programas de formación respondan a la demanda de la sociedad misma. En esos mismos términos se manifiesta Carrión (2003), cuando argumenta que el rol de la educación superior es formar profesionales universitarios para resolver los problemas que aquejan a la población. Lo anterior constituye una crítica al sistema educativo Latinoamericano, en donde es incipiente la relación de la universidad con los sectores productivos de la sociedad, aspecto que en los países desarrollados ya se da por superado (Plata 2003).

En la Conferencia Regional de Educación Superior en América Latina y el Caribe 2008 la pertinencia de la educación superior también fue ampliamente discutida y debatida, concluyéndose que en efecto falta camino por recorrer en ese sentido, pero existe consenso sobre la necesidad de avanzar en esa dirección (UNESCO 2008).

El 68\% de las personas consultadas considera que la educación universitaria de Honduras en relación a 
SAN es poco pertinente. Sólo $12 \%$ la considera muy pertinente y en esa misma proporción se ubica la calificación de bastante y nada pertinente. La percepción de poco y nada pertinente, es más generalizada entre los encuestados de ONG y la cooperación internacional; el 83\% de los primeros y el 92\% de los segundos tienen esa opinión (Figura 1).

Es probable que esa percepción de poca pertinencia sea un indicador de que la SAN no está siendo abordada en carreras universitarias relacionadas con la disponibilidad, acceso, consumo y utilización de los alimentos. Son diversos los campos profesionales que tienen esa relación, pero es más evidente y aceptado por la mayoría, la vinculación de la SAN con los estudios de agronomía, medicina, nutrición humana, procesamiento de alimentos y todas aquellas carreras que contemplen formación en planificación y desarrollo, especialmente del sector rural.

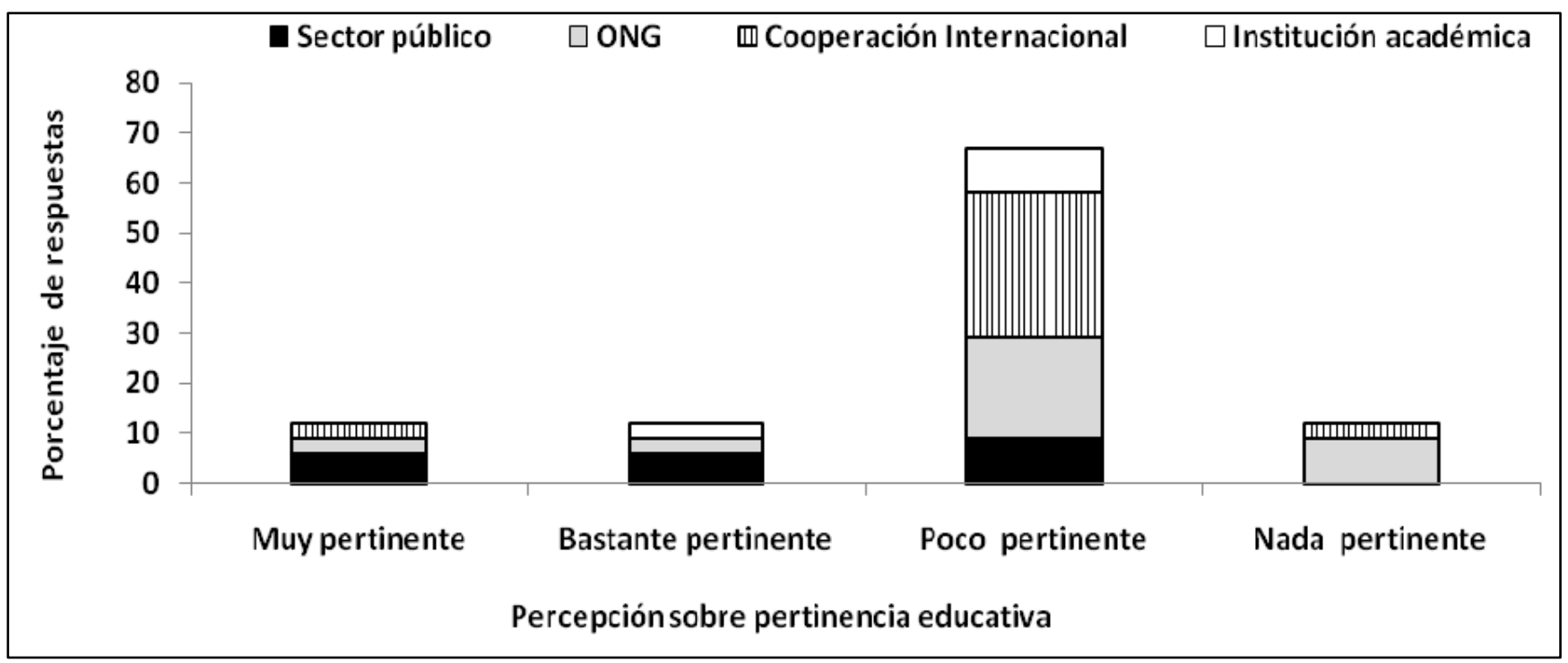

Figura 1. Percepción por sector profesional sobre pertinencia de la formación universitaria en relación a la Seguridad Alimentaria Nacional.

Oferta de formación en SAN. En 2004, el Consejo Superior Universitario de Centroamérica (CSUCA) con apoyo de diversas entidades como FAO y el Instituto de Nutrición de Centroamérica y Panamá (INCAP), elaboró y aprobó el Plan de Acción Universitario en Seguridad Alimentaria y Nutricional (PAUSAN). Dicho plan define el involucramiento de las universidades públicas de la región en procesos de formación, investigación y extensión en SAN. La operatividad del PAUSAN contempla una serie de acciones tendientes a sensibilizar a la comunidad universitaria sobre la importancia de la SAN, incluir la temática en el currículo de diversas carreras universitarias, creación de programas especiales a nivel de pre y post-grado, promoción de investigaciones y capacitación de cuadros técnicos de gobiernos y ONG (CSUCA et al. 2004).

Actualmente las experiencias más desarrolladas a nivel de postgrado son la Maestría en Alimentación y Nutrición (MANA) y la Maestría en SAN y Desarrollo Local, ofertadas por la Universidad de San Carlos (USAC) en Guatemala. Otro programa novedoso es el programa de Maestría en Gerencia en SAN, promovido por la Escuela de Ciencias Agrarias de la Universidad Nacional de Costa Rica y la Escuela de Nutrición de la Universidad de Costa Rica. En Nicaragua se ha dado un buen ejemplo de cooperación interuniversitaria, la Universidad Nacional Autónoma de Nicaragua-León, la Universidad Centroamericana (UCA) y la Universidad Nacional Agraria (UNA) han 
promovido un postgrado en SAN cuyo objetivo es capacitar los cuadros técnicos del gobierno y de ONG, así como docentes de las mismas universidades involucradas. Una característica común de estos programas es que han sido creados con el apoyo de la cooperación internacional, donde destaca la FAO a través de sus diversos programas en la región y el INCAP como centro regional especializado en alimentación y nutrición. Otras universidades de la región han desarrollado cursos de actualización, pero los mismos no han evolucionado a programas formales que conduzcan a la obtención de algún grado académico.

Los programas de formación en SAN a nivel de postgrado en Centroamérica son poco conocidos entre las personas consultadas en Honduras, sólo 34\% de ellos manifestó tener algún conocimiento de los mismos, destacando los programas que apoya el INCAP en Guatemala como los más conocidos. El estudio también confirma que después de cinco años del establecimiento del PAUSAN, los resultados en Honduras son mínimos; la oferta de formación en SAN a nivel de pre y postgrado es considerada por una amplia mayoría (90\%) entre baja y/o nula. Sólo 50\% de las personas consultadas manifestaron conocer un programa de formación a nivel de pregrado, refiriéndose en su totalidad al Profesorado en Educación en SAN de la Universidad Pedagógica Nacional Francisco Morazán (UPNFM). El 74\% de los que manifestaron conocer el citado programa, lo califican como de regular y/o mala calidad; algunas personas manifestaron la urgencia de una revisión y evaluación profunda del programa para adecuarlo a las exigencias de la sociedad.

Se pidió a los encuestados sugerir acciones para mejorar la situación actual, producto de esa consulta surgen dos opciones; aumentar la oferta de programas (68\%) y mejorar los programas existentes (32\%). Entre los que proponen una mejora de los programas existentes, predomina la sugerencia de que la SAN sea abordada con una mayor contundencia y especificidad en los programas de educación agrícola; una posición muy acorde con la propuesta de los organismos internacionales de apoyar la agricultura como elemento indispensable para combatir el hambre en el mundo. En Honduras, la formación agrícola está presente en tres niveles: superior, medio y no formal. En el nivel superior existen seis centros universitarios que ofrecen la carrera de agronomía, a nivel medio existen tres escuelas agrícolas regionales y el nivel no formal está a cargo del Instituto Nacional de Formación Profesional (Ávila et al. 2002). En la actualidad, la mayoría de ellos abordan la temática del desarrollo rural; pero no se cuenta con programas específicos de formación en seguridad alimentaria nutricional, aunque es probable que en algunos casos el tema sea abordado como parte de una temática más amplia.

A los que propusieron aumentar la oferta de programas específicos en SAN, se les pidió priorizar, utilizando una escala cualitativa de mayor a menor importancia entre diferentes niveles de formación: postgrado, pregrado, diplomado y capacitaciones cortas (seminarios y talleres). Es evidente la mayor importancia del nivel de pregrado (Cuadro 3 y Figura 2 ), posiblemente porque en las organizaciones el perfil más requerido es personal técnico. Esta inquietud debe ser considerada por los centros universitarios del país, ya que indica una demanda de formación no satisfecha. Es probable que los programas de diplomados y capacitaciones cortas tengan menos importancia porque ese tipo de formación se cubre por las mismas organizaciones, que desarrollan eventos para actualizar conocimientos de su personal. A pesar de su inexistencia en Honduras, los expertos asignan el mismo grado de importancia a los postgrados que a las capacitaciones cortas (Figura 2).

Un aspecto a considerar en los programas académicos en SAN (nuevos o mejorados) es la diversidad de criterios que se manejan alrededor del mismo concepto. En los países desarrollados referirse a Seguridad Alimentaria es abordar básicamente lo concerniente a la calidad e inocuidad de los alimentos y esa es la principal preocupación de sus ciudadanos (González 2003). En cambio, en los países en vías de desarrollo, el mismo término hace mayor referencia a la disponibilidad de alimentos para la población, ya que esa es la principal limitante. Seguridad alimentaria incluye ambos elementos, pero es muy importante entender las prioridades y enfoques en cada región para adecuar los programas de formación a esa realidad específica. Otros conceptos relacionados, como soberanía alimentaria y derecho a la alimentación, también son muy importantes y deben ser parte de los programas académicos. 
Cuadro 3. Número de respuestas en cada nivel de formación.

\begin{tabular}{lcccc}
\hline & $\begin{array}{c}\mathbf{1} \\
\text { Extremadamente } \\
\text { importante }\end{array}$ & $\begin{array}{c}\mathbf{2} \\
\text { Muy importante }\end{array}$ & $\begin{array}{c}\mathbf{3} \\
\text { Bastante } \\
\text { importante }\end{array}$ & $\begin{array}{c}\mathbf{4} \\
\text { Poco importante }\end{array}$ \\
\hline Postgrado & 11 & 13 & 9 & 22 \\
Pregrado & 25 & 19 & 11 & 0 \\
Diplomados & 10 & 17 & 11 & 17 \\
Capacitaciones cortas & 9 & 6 & 24 & 16 \\
\hline
\end{tabular}

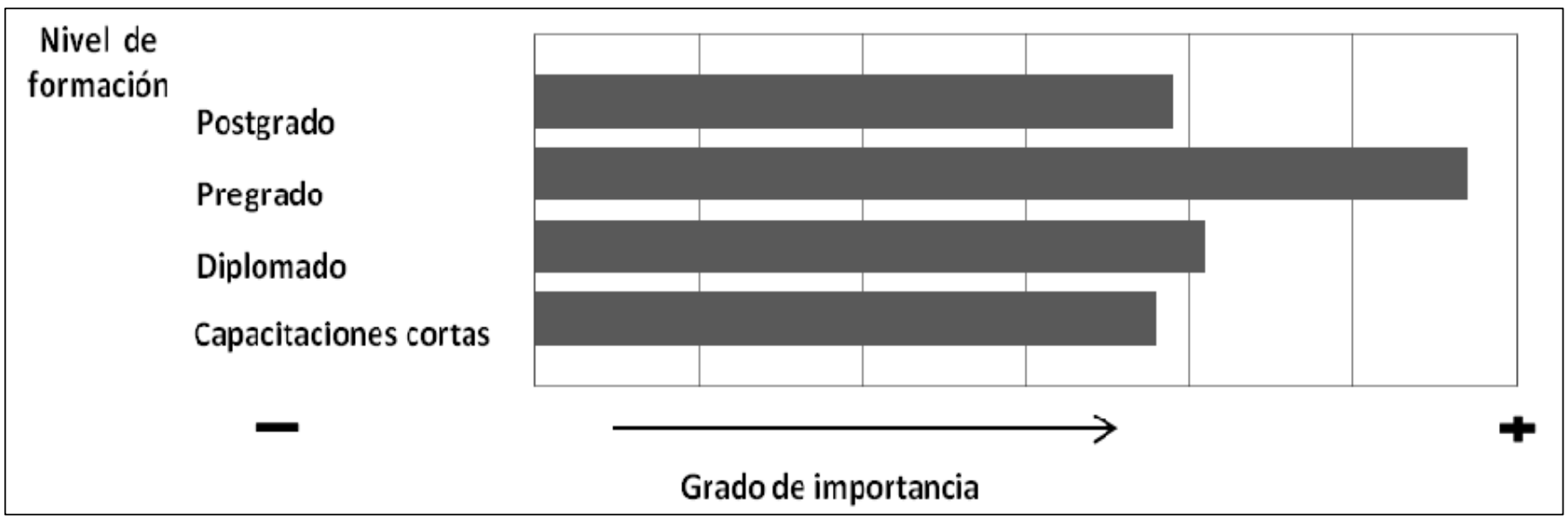

Figura 2. Grado de importancia de cuatro niveles de formación en Seguridad Alimentaria Nacional.

Formación en competencias. Aunque la formación en competencias es un concepto antiguo, en la educación superior ha tenido su mayor impulso en los últimos 15 años; una de las razones es su probada eficacia para brindar una formación con mayor integralidad (Alvarez 2004). La formación en competencias en la educación superior tiene el objetivo de acercar la universidad a la sociedad y proporcionar una enseñanza más práctica y útil para los estudiantes (Yanis 2008). Existen diversos modelos de formación en competencias, uno de ellos es el propuesto por el International Project Management Association (IPMA) y por la Asociación Española de Ingeniería de Proyectos (AEIPRO). Este modelo propone la formación en base a 46 elementos de competencia específicos para la dirección de proyectos, agrupados en tres categorías: técnicas (20), comportamiento (15) y contextuales (11) (AEIPRO e IPMA 2006). Esa característica lo convierte en una excelente opción para los programas de formación en seguridad alimentaria, ya que la mayoría de acciones en los territorios más desfavorecidos se desarrollan mediante programas y proyectos de corto, mediano y largo plazo.

Previo a la inclusión del tema de formación en competencias en la encuesta, mediante un sondeo entre alumnos del Doctorado de Planificación y Gestión de Proyectos de Desarrollo Rural Sostenible de la Universidad Politécnica de Madrid, se seleccionaron los siguientes 15 elementos de competencia (cinco de cada grupo) que se consideran claves para un director de proyectos:

Competencias técnicas: información y documentación, dirección de proyectos, trabajo en equipo, resolución de problemas y comunicación.

Competencias de comportamiento: liderazgo, actitud abierta, creatividad, negociación y ética.

Competencias contextuales: relaciones con organizaciones, implantación de nuevas tecnologías, dirección de personal, responsabilidad con el medio 
Ardón, C.R. y Afonso Gallegos, A.: Percepciones sobre programas de formación e institucionalidad en Seguridad Alimentaria

ambiente y entendimiento del marco legal y político.

En la encuesta se pidió a los participantes que entre los 15 elementos de competencia proporcionados, marcaran aquellos que a su criterio deberían ser abordados obligatoriamente en los programas de formación en SAN; la valoración se realizó en base al número de marcas obtenidas por cada uno en relación al total (Figura 3). Dentro de las competencias técnicas destacan la dirección de proyectos y la comunicación. En las competencias de comportamiento, que son muy útiles para relacionarse con los diferentes actores, conceden mayor relevancia al liderazgo. Entre las competencias contextuales que son básicas para entender el entorno, es evidente la mayor importancia asignada a las relaciones con organizaciones, entendimiento del marco legal y político, y la responsabilidad con el medio ambiente.

Por lo menos tres elementos de competencia de cada grupo se ubican por arriba del $60 \%$, lo cual indica que las personas consultadas valoran mucho la integralidad en los programas de formación. Tres elementos de competencia contextuales se encuentran entre los cinco más importantes, un dato muy significativo, contrario al modelo anterior donde se pensaba que los programas debían centrarse en desarrollar competencias técnicas, olvidándose que un buen profesional también debe ser capaz de entender el entorno y relacionarse con los demás.

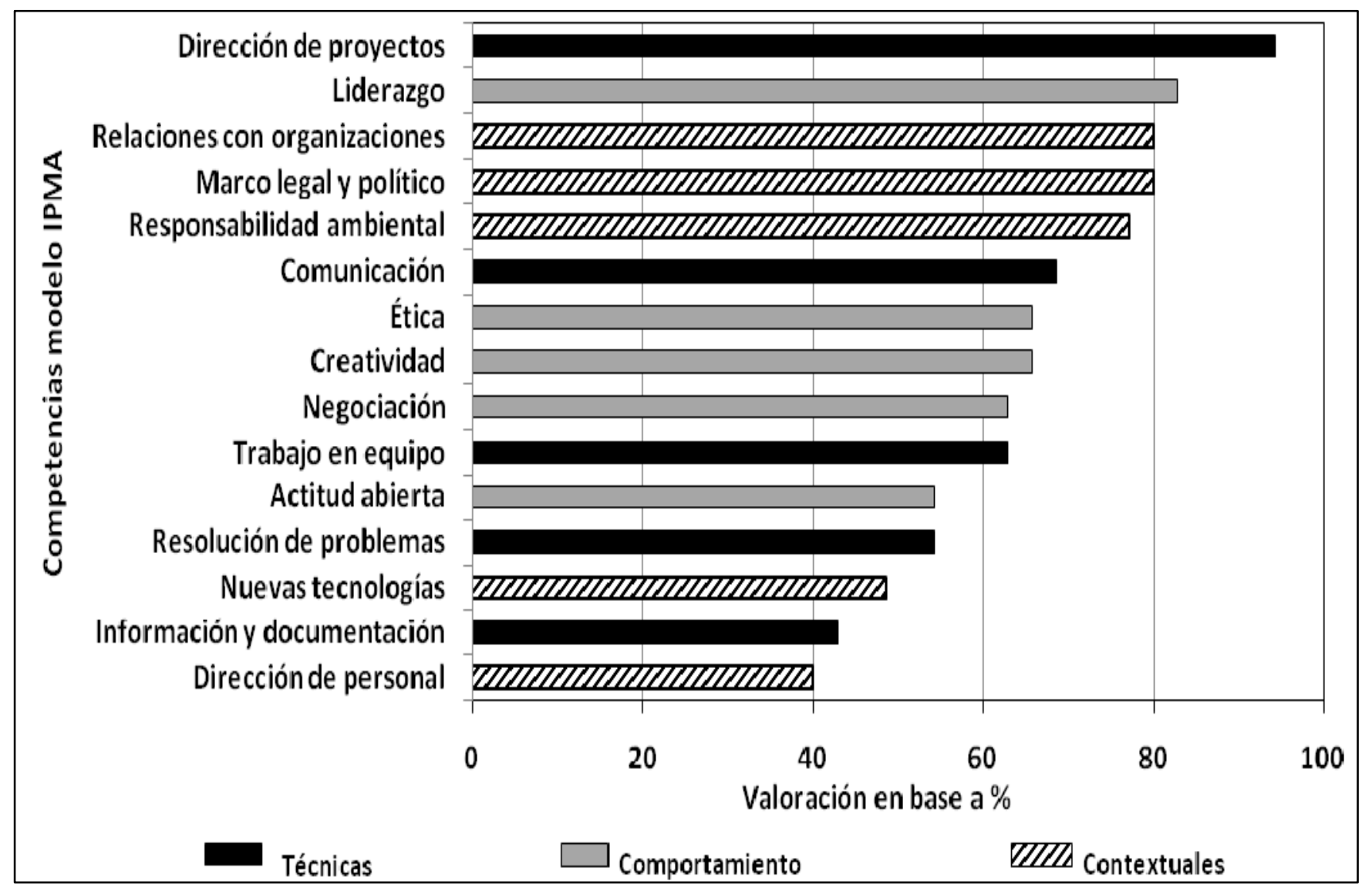

Figura 3. Valoración asignada a elementos de competencia del modelo IPMA (International Project Management Association). 


\section{Institucionalidad SAN en Honduras y rol de los actores}

Presencia y conocimiento de instancias de coordinación. A nivel gubernamental, la lucha contra el hambre en Honduras está inserta en la Estrategia de Reducción de la Pobreza (ERP). La instancia superior es el Gabinete Social, presidido por el presidente de la República e integrado por diferentes Secretarías de Estado. Al mismo nivel está conformado el Consejo Consultivo de la ERP, que está integrado por representantes de las organizaciones de la sociedad civil, empresa privada y gobiernos locales, así como la cooperación internacional; su función es asesorar al Gabinete Social en todo lo relacionado a la implementación y seguimiento de la ERP (República de Honduras 2006). A pesar de estar involucradas según mandato, en la práctica la participación de la sociedad civil en la ERP es limitada, teniendo poca participación en las decisiones importantes (Dijkstra 2007). Hay otras instancias en donde la sociedad civil y las ONG vinculadas a la seguridad alimentaria tienen mayor participación, dentro de estas instancias están el Comité Técnico Interinstitucional de SAN (COTISAN), la Coalición SAN, y la Alianza para la Soberanía y Seguridad Alimentaria en Honduras (SARA).

El COTISAN surge como parte de los acuerdos para la implementación de la Política de Seguridad
Alimentaria y Nutricional y es coordinado por la Unidad Técnica de SAN (UNAT-SAN) de la Secretaría del Despacho Presidencial. Sus objetivos son apoyar la implementación, actualización y revisión de las políticas de SAN y establecer un espacio de coordinación de acciones e intercambio de experiencias para el logro de las metas en SAN definidas por el país (UNAT-SAN 2009). La Coalición SAN es otra instancia en donde convergen las organizaciones que trabajan en SAN en Honduras; especialmente aquellas organizaciones no gubernamentales apoyadas por la cooperación internacional y que ejecutan programas y proyectos en distintas regiones del territorio hondureño. Surge por interés de diversas organizaciones para establecer mecanismos de coordinación, intercambio e incidencia en políticas. Otro punto de encuentro sobre la SAN es la Alianza para la Soberanía y Seguridad Alimentaria en Honduras (SARA), creada en febrero de 2007 y cuyo fin es incidir a nivel gubernamental para llevar a cabo procesos eficientes de reforma agraria asociada a la soberanía alimentaria. SARA pertenece a la Red Centroamericana de Seguridad y Soberanía Alimentaria (RedCassan) y de esa manera está articulada con el resto de organizaciones a nivel mundial como la International Food Security Network (IFSN). En el cuadro 4 se detalla el tipo y número de organizaciones que participan en cada una de estas instancias de coordinación.

Cuadro 4. Tipo y número de organizaciones que conforman las instancias de coordinación.

\begin{tabular}{lccc}
\hline Sectores representados & $\begin{array}{c}\text { Coalición SAN } \\
\text { (directorio 2007) }\end{array}$ & $\begin{array}{c}\text { COTISAN } \\
\text { (directorio 2008) }\end{array}$ & $\begin{array}{c}\text { SARA } \\
\text { (directorio 2008) }\end{array}$ \\
\hline Sector público & 8 & 10 & 0 \\
ONG & 21 & 7 & 7 \\
Cooperación Internacional & 11 & 4 & 0 \\
Universidades & 4 & 0 & 0 \\
Sociedad civil organizada & 0 & 0 & 8 \\
Total & $\mathbf{4 4}$ & $\mathbf{2 1}$ & $\mathbf{1 5}$ \\
\hline
\end{tabular}

Los expertos en quienes estaba enfocada la consulta pertenecen o han participado en alguna de estas instancias y es lógico que estén enterados de su funcionamiento, pero con el fin de conocer su nivel de integración, se consideró importante determinar el grado de conocimiento de cada uno de ellos sobre las demás organizaciones. Se percibe un conocimiento alto de COTISAN y Coalición SAN, ya que $89 \%$ manifestó conocer una o ambas. Sólo 14\% manifestó conocer la organización SARA, algo preocupante porque esta instancia está conformada por organizaciones de la sociedad civil que aglutinan a los 
colectivos con mayores problemas de seguridad alimentaria y se esperaría mayor conocimiento de la misma de miembros del gobierno y de las ONG. Algunos encuestados manifestaron la existencia de dos organizaciones más, la Alianza Nacional contra el Hambre (ANCHA) y la Red de SAN del Occidente de Honduras (SAN-ROH). La primera fue creada a instancias del sector gubernamental en 2003 y es coordinada por la Secretaría de Agricultura, pero no hay evidencia de que en la actualidad se mantenga activa. La segunda es una red local de instituciones en el occidente de Hondura, considerada la zona con mayores niveles de pobreza en el territorio nacional.

Beneficios percibidos de pertenecer a foros sobre $\underline{\text { SAN. Según Hoyos (2003), los procesos participativos }}$ son muy importantes para dar legitimidad a las decisiones, si los ciudadanos no participan en las decisiones que les afectan y en la definición de leyes que regulan su comportamiento, difícilmente dedicarán esfuerzos para la búsqueda de soluciones. Desgraciadamente, esa es una situación muy común en las sociedades actuales. A nivel de organizaciones de desarrollo la situación es similar, la participación en instancias de coordinación es escasa porque muchas veces éstas se convierten en espacios poco productivos. Con el fin de conocer la percepción sobre los beneficios derivados de la participación en las diferentes instancias de coordinación, se seleccionaron cinco posibles beneficios y se pidió valorar la importancia de cada uno de ellos mediante una escala cualitativa de mayor a menor importancia. Los beneficios propuestos para valoración fueron seleccionados de acuerdo a los objetivos planteados por cada una de las instancias y reflejan de alguna manera las razones de su conformación y funcionamiento (Cuadro 5).

Cuadro 5. Número de respuestas en cada beneficio de participar en foros sobre Seguridad Alimentaria Nacional (SAN).

\begin{tabular}{|c|c|c|c|c|c|}
\hline $\begin{array}{l}\text { Beneficios de participar } \\
\text { en foros sobre SAN }\end{array}$ & $\begin{array}{c}1 \\
\begin{array}{c}\text { Extremadamente } \\
\text { importante }\end{array}\end{array}$ & $\begin{array}{c}2 \\
\text { Muy } \\
\text { importante }\end{array}$ & $\begin{array}{c}3 \\
\text { Bastante } \\
\text { Importante }\end{array}$ & $\begin{array}{c}4 \\
\begin{array}{c}\text { Medianamente } \\
\text { importante }\end{array}\end{array}$ & $\begin{array}{c}5 \\
\text { Poco } \\
\text { Importante } \\
\end{array}$ \\
\hline $\begin{array}{l}\text { Intercambio de } \\
\text { información }\end{array}$ & 16 & 9 & 13 & 9 & 8 \\
\hline $\begin{array}{l}\text { Elaboración de planes de } \\
\text { acción conjuntos }\end{array}$ & 13 & 14 & 8 & 17 & 3 \\
\hline Capacitación de personal & 6 & 9 & 14 & 13 & 13 \\
\hline $\begin{array}{l}\text { Coordinación de acciones } \\
\text { en los territorios }\end{array}$ & 6 & 9 & 13 & 11 & 16 \\
\hline $\begin{array}{l}\text { Incidencia en políticas } \\
\text { públicas }\end{array}$ & 2 & 2 & 8 & 2 & 41 \\
\hline
\end{tabular}

La figura 4 elaborada con los datos del cuadro 5 , refleja que el intercambio de información y la elaboración de planes de acción conjuntos son los beneficios más valorados; la capacitación de personal y la coordinación de acciones en los territorios medianamente valorados y la incidencia en políticas públicas el beneficio menos valorado. Este aspecto debe llamar la atención a los integrantes de dichos foros, porque la incidencia en políticas es justamente uno de los objetivos más destacados de su conformación y no es percibido como un beneficio tangible por sus integrantes.

Participación de universidades en foros sobre SAN. Ya se comentó que la participación de las universidades en los procesos de desarrollo es determinante para el desarrollo de un país. Silva (2005) destaca que la universidad, además de la formación de profesionales y el desarrollo científico, debe comprometerse con la solución de los problemas que aquejan a la sociedad 
actual, contribuyendo de esa manera al desarrollo económico y social. En relación a ese tema y mediante una escala cualitativa se pidió valorar la participación de las universidades hondureñas en los foros de coordinación listados anteriormente. El resultado indica que dicha participación es considerada poco relevante por $67 \%$ de los encuestados; sólo un poco más de $10 \%$ la considera muy relevante (Figura 5). Es interesante que todos los que pertenecen a instituciones educativas asignan una calificación entre poco y nada relevante. Este resultado refleja una situación preocupante, pero a la vez de oportunidad para las universidades, porque significa que hay un espacio para incorporarse y aportar a la solución de un problema de interés nacional. El mismo tiene mucha relación con la percepción de pertinencia; de hecho, la poca participación de las universidades en los foros, con toda probabilidad contribuye a la percepción de poca pertinencia de la educación superior en relación a la SAN en Honduras.

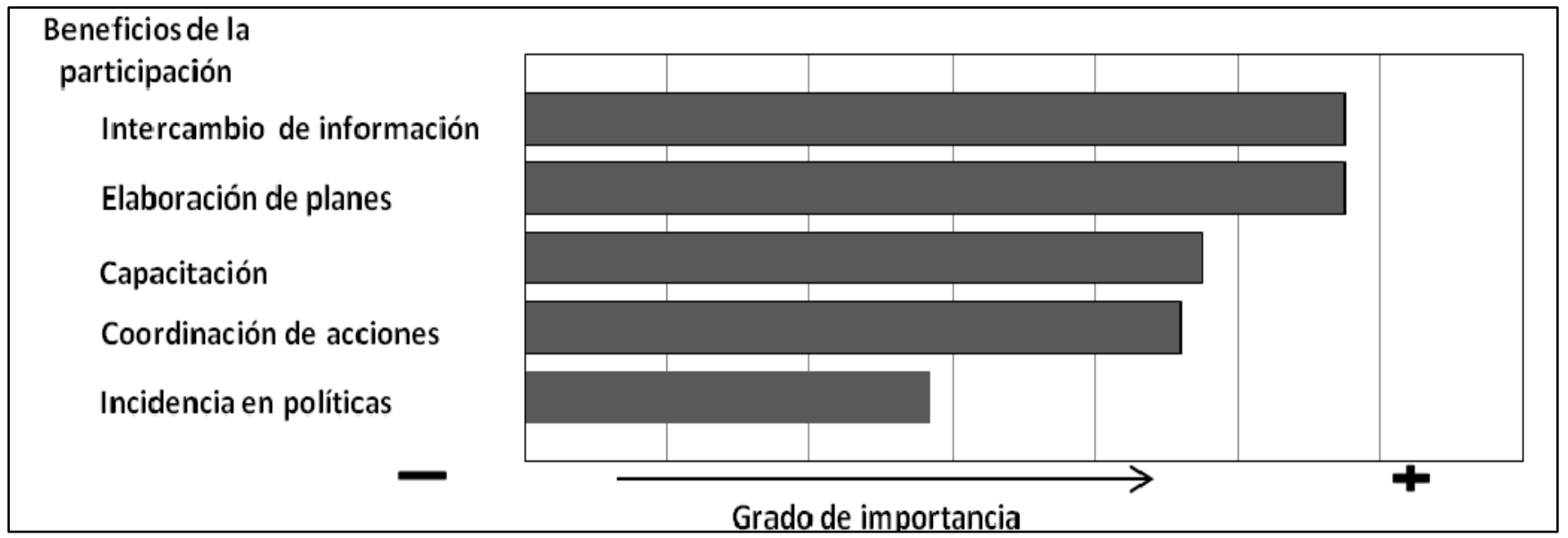

Figura 4. Grado de importancia de beneficios de pertenecer a foros sobre Seguridad Alimentaria Nacional.

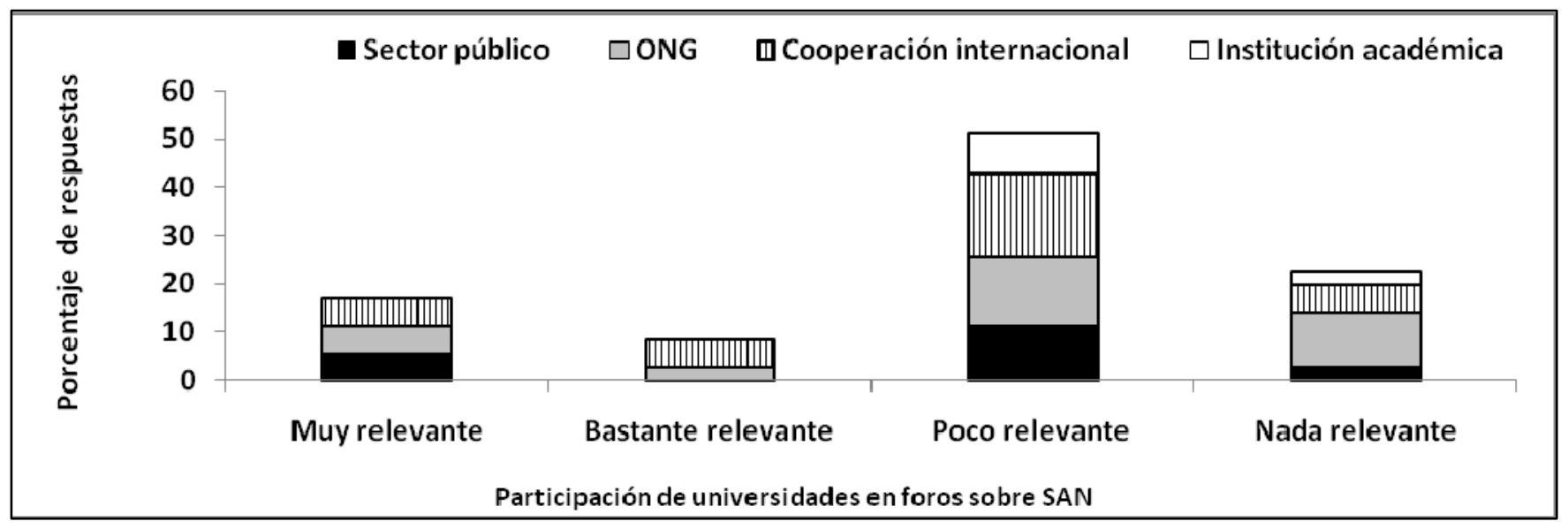

Figura 5. Percepción sobre participación de universidades en foros de Seguridad Alimentaria Nacional (SAN). 
Generación de información sobre SAN. La información de calidad es determinante para la toma de decisiones acertadas. La gestión de información es el proceso por el cual se recolecta, organiza y se distribuye la información a los usuarios; un proceso clave para enfrentar los grandes problemas de la sociedad (Bonilla 2003). Con base en este argumento, se consultó sobre la cantidad y calidad de información sobre la SAN generada en Honduras; asignando en una escala cualitativa las valoraciones. El $86 \%$ opina que es poca la información generada, sólo $14 \%$ cree que se produce información en alta y muy alta cantidad. En la calidad, 69\% cree que la información generada es de regular calidad y $28 \%$ la ubica entre buena y excelente.

La percepción de cantidad y calidad de información dependerá de la demanda de cada uno. Un comentario generalizado en la sección de observaciones de la encuesta, es que se dispone de información sobre indicadores a nivel macro; pero se carece de información específica sobre el estado de la población en los territorios donde el problema está presente y donde es prioritario combatir la inseguridad alimentaria. Independientemente de los factores que hayan influido la respuesta, esta combinación de resultados negativos (poca información y de regular calidad), hace necesario que se definan responsabilidades para suplir esa carencia y contar con la información demandada por cada sector.

Existirán diversas razones que justifiquen la calificación de poca generación de información; a los que asignaron esa calificación se les solicitó valorar mediante una escala cualitativa de mayor a menor importancia las siguientes cinco causas posibles: ausencia de centros de investigación, falta de profesionales para hacer investigación, falta de recursos financieros para la investigación y limitada demanda de información por parte de las mismas organizaciones (Cuadro 6 y Figura 6).

Cuadro 6. Número de respuestas en cada razón de poca generación de información.

\begin{tabular}{lcccc}
\hline $\begin{array}{l}\text { Razones de poca generación de } \\
\text { información }\end{array}$ & $\begin{array}{c}\mathbf{1} \\
\text { Extremadamente } \\
\text { importante }\end{array}$ & $\begin{array}{c}\mathbf{2} \\
\text { Muy } \\
\text { importante }\end{array}$ & $\begin{array}{c}\mathbf{3} \\
\text { Bastante } \\
\text { importante }\end{array}$ & $\begin{array}{c}\mathbf{4} \\
\text { Poco } \\
\text { importante }\end{array}$ \\
\hline $\begin{array}{l}\text { Limitada demanda de información por las } \\
\text { organizaciones }\end{array}$ & 9 & 12 & 6 & 28 \\
$\begin{array}{l}\text { Falta de profesionales } \\
\text { Ausencia de centros de investigación }\end{array}$ & 12 & 17 & 21 & 5 \\
Falta de recursos financieros & 15 & 17 & 14 & 9 \\
\hline
\end{tabular}

\section{Razones de poca \\ generación de información}

Ausencia de centros de investigación

Falta de profesionales

Falta de recursos financieros

Limitada demanda

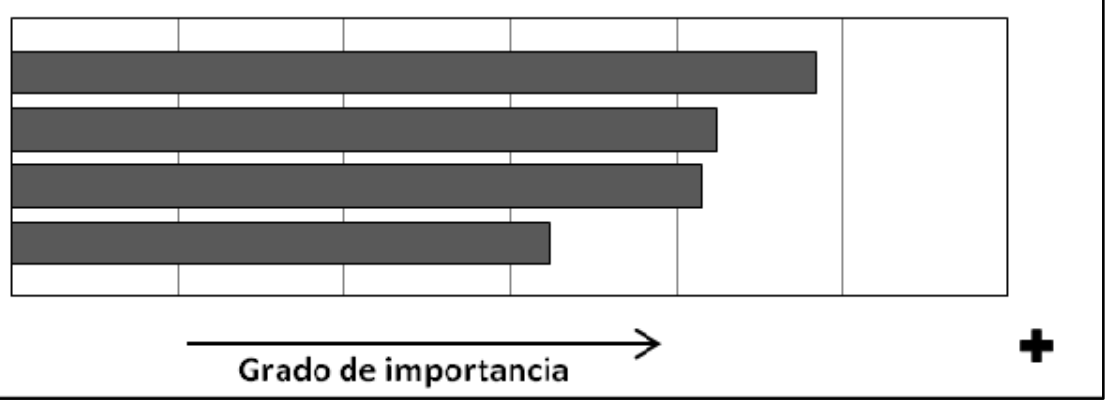

Grado de importancia

Figura 6. Grado de importancia de las razones de la poca generación de información sobre Seguridad Alimentaria Nacional. 
Se percibe que la poca disponibilidad de información se debe en mayor medida a la ausencia de centros de investigación, falta de profesionales y falta de recursos financieros; y en menor medida a la limitada demanda por las organizaciones (Figura 6). Otra manera de entender este resultado es que las organizaciones demandan la información, pero debido a los otros factores dicha información no está disponible. La ausencia de centros de investigación está muy en consonancia con la poca participación de las universidades en los foros y la poca pertinencia de la educación superior detallada en apartados anteriores. Aunque en la priorización de niveles de formación fue más valorado el nivel de pregrado, considerar la falta de profesionales como una causa importante de la poca generación de información, refleja que es necesario también mejorar la oferta de postgrado, ya que en este nivel es donde se fortalecen más las capacidades investigativas de los profesionales.

Con el objetivo de conocer quienes generan la información sobre la SAN en Honduras y quienes deberían generarla, se pidió a los participantes asignar una priorización en ese sentido, haciendo uso de una escala cualitativa de mayor a menor importancia y considerando los sectores que normalmente participan en este tipo de función: sector público, ONG, cooperación internacional y universidades (Cuadro 7). Valorando estos resultados, se deduce que la cooperación internacional y las ONG son las que generan más información (Figura 7). Por el contrario, el sector público y las universidades las que generan menos información.

Cuadro 7. Número de respuestas en cada sector de acuerdo a responsabilidad actual de generar información Seguridad Alimentaria Nacional.

\begin{tabular}{lcccc}
\hline & $\begin{array}{c}\mathbf{1} \\
\text { Extremadamente } \\
\text { importante }\end{array}$ & $\begin{array}{c}\mathbf{2} \\
\text { Muy importante }\end{array}$ & $\begin{array}{c}\mathbf{3} \\
\text { Bastante } \\
\text { importante }\end{array}$ & $\begin{array}{c}\mathbf{4} \\
\text { Poco importante }\end{array}$ \\
\hline Sector público & 6 & 13 & 23 & 13 \\
ONG & 20 & 25 & 8 & 2 \\
Universidades & 0 & 3 & 13 & 39 \\
Cooperación internacional & 31 & 14 & 8 & 2 \\
\hline
\end{tabular}

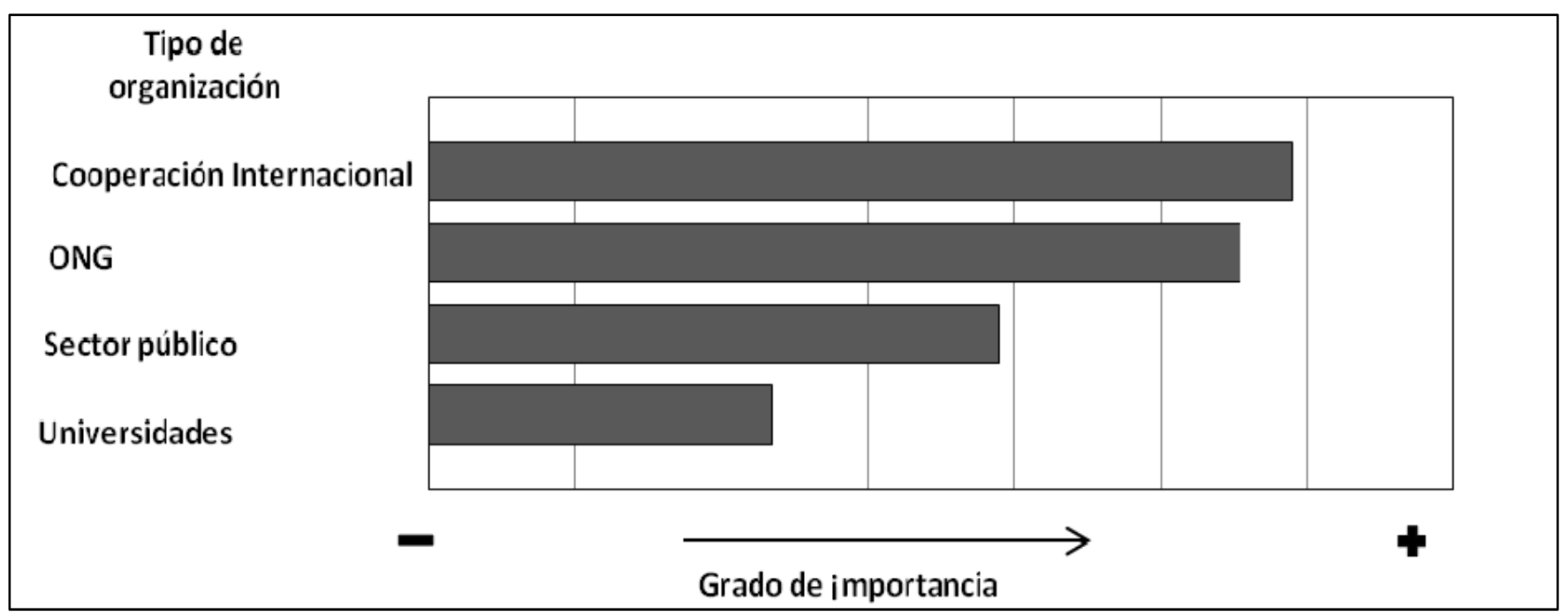

Figura 7. Grado de responsabilidad actual en generar información sobre Seguridad Alimentaria Nacional. 
Ardón, C.R. y Afonso Gallegos, A.: Percepciones sobre programas de formación e institucionalidad en Seguridad Alimentaria

Al preguntar sobre la situación ideal, los resultados que se obtienen son diferentes. Se cree que el sector público y las universidades deberían tener mayor responsabilidad en la generación de información y menos responsabilidad las ONG y la cooperación internacional (Cuadro 8 y Figura 8). Esta percepción de cambio es muy lógica, ya que el sector público es quien debe liderar la toma de decisiones y es en las universidades donde están los investigadores con capacidad de generar conocimientos y deberían estar más involucrados en estos procesos. Sin embargo, existe la limitante financiera; el gobierno y las universidades, por lo general, no disponen de recursos suficientes y la generación de información no entra en sus prioridades. Quiñones y Alvarez (2004) destacan que justamente la función de investigación y transmisión de conocimientos es la menos desarrollada en las universidades y constituye una seria crítica de la sociedad; argumentan también que la investigación es un problema complejo y para su desarrollo es necesario, además de la voluntad de los docentes y estudiantes, disponer de los recursos técnicos y científicos adecuados. FAO y UNESCO (2003) confirman este hecho al afirmar que, aunque en América Latina ha aumentado la oferta de postgrados en las universidades, estos tienen poca relación con la investigación y la transferencia de conocimientos a otros sectores de la sociedad.

Cuadro 8. Número de respuestas en cada sector de acuerdo a responsabilidad sugerida de generar información Seguridad Alimentaria Nacional (SAN).

\begin{tabular}{lcccc}
\hline Sector & $\begin{array}{c}\mathbf{1} \\
\text { Extremadamente } \\
\text { importante }\end{array}$ & $\begin{array}{c}\mathbf{2} \\
\text { Muy } \\
\text { importante }\end{array}$ & $\begin{array}{c}\mathbf{3} \\
\text { Bastante } \\
\text { importante }\end{array}$ & $\begin{array}{c}\mathbf{4} \\
\text { Poco } \\
\text { importante }\end{array}$ \\
\hline Sector público & 35 & 17 & 3 & 0 \\
ONG & 3 & 9 & 36 & 6 \\
Universidades & 20 & 27 & 3 & 5 \\
Cooperación internacional & 0 & 0 & 11 & 44 \\
\hline
\end{tabular}

Tipo de organización

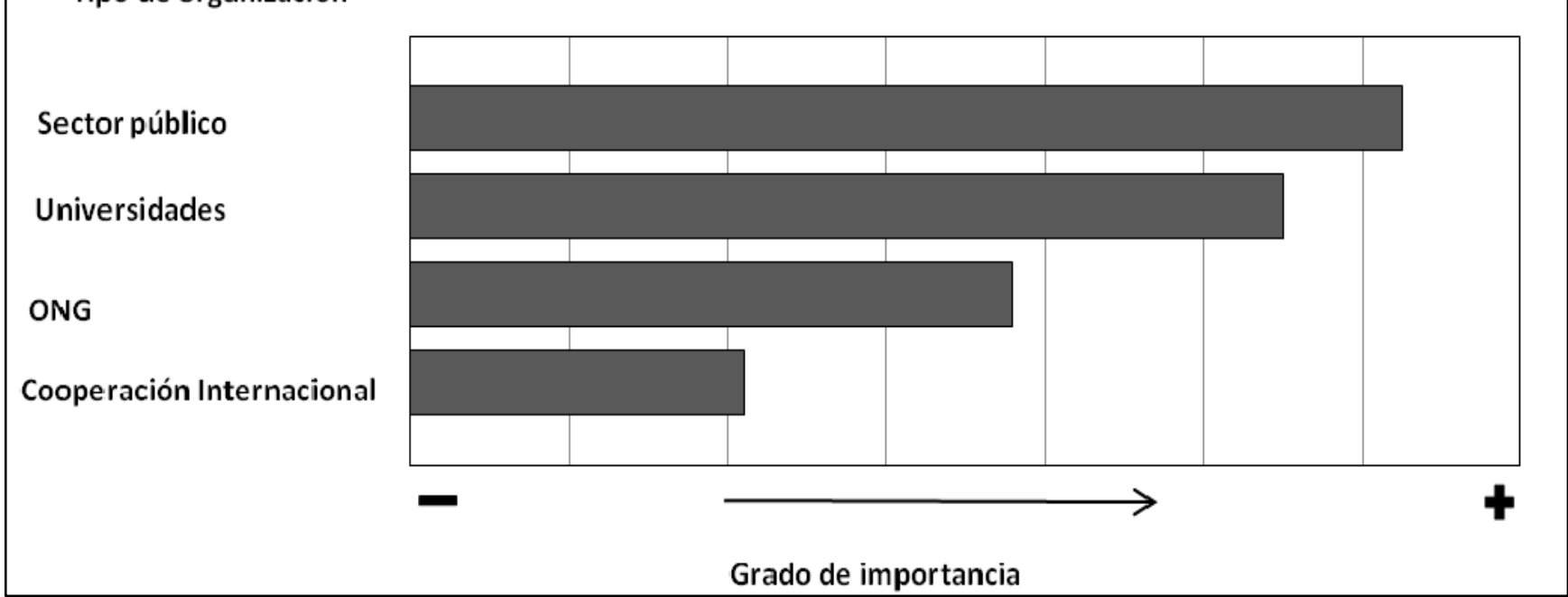

Figura 8. Grado de responsabilidad sugerida para generar información sobre Seguridad Alimentaria Nacional (SAN). 
Considerando que una de las causas de inconformidad con la situación actual es la falta de información específica sobre los territorios más desfavorecidos, las universidades podrían suplir esa carencia, enfocando los trabajos de graduación (tesis, monografías, etc.) de sus estudiantes hacia esa necesidad en particular. Según Oseguera (2005) aunque la investigación universitaria en Honduras es menor que en otros países, hay experiencias interesantes de generación de conocimientos, especialmente de universidades dedicadas a la formación en ciencias agrícolas que promueven investigaciones en mejoramiento de cultivos y manejo de los mismos. Seguramente en otras áreas como medicina, nutrición humana, manejo de alimentos, también se genere información relevante, pero si la misma no es enfocada desde la perspectiva de la SAN, no es valorado su aporte a la solución del problema.

Es importante recordar que la inseguridad alimentaria depende de diversidad de factores y aunque no existan programas específicos de formación en SAN, cualquier carrera universitaria puede colaborar en estudios que permitan conocer mejor la realidad para tomar las decisiones más acertadas.

Marco de políticas SAN. Un aspecto clave en la lucha contra el hambre es la voluntad política de los estados, traducida por supuesto en políticas públicas adecuadas y programas de apoyo a los colectivos con mayores problemas. De acuerdo a Sánchez (1993), en la definición de políticas juega un rol determinante el conocimiento que se tenga de la realidad. Si se pretende elaborar políticas eficientes que conduzcan a la solución de determinado problema, es preciso hacer diagnósticos detallados para contar con información de calidad. Afortunadamente, y gracias a la participación de los sectores sociales, se ha evolucionado bastante en ese sentido.

En los últimos años y con participación de esos sectores sociales, en Honduras se ha promovido una serie de políticas relacionadas con la SAN; en ellas se da bastante relevancia al sector rural, por ser donde la problemática de seguridad alimentaria es mayor, pero también donde se pueden generar soluciones. El marco de políticas también hace referencia a la coordinación intersectorial, motivando la promoción de proyectos conjuntos que garanticen un mayor impacto en la población beneficiaria. En el cuadro 9 se resumen las principales políticas y los elementos claves en cada una de ellas.

En la encuesta se solicitó a las personas opinión sobre este marco de políticas, el 43\% percibe que es suficiente, pero es necesario mayor compromiso de todos los actores para su cumplimiento; destacan como aspectos a mejorar la definición clara de responsabilidades y una mejor coordinación entre las dependencias del estado. El resto de encuestados que argumentan insuficiencia en las políticas (56\%), hacen referencia a la carencia de políticas sobre derecho a la alimentación, un elemento presente en la agenda de la iniciativa América Latina y El Caribe sin hambre que impulsa la FAO en la región y que está siendo discutido y promovido en casi todos los estados miembros (ALCSH 2009).

\section{Conclusiones y Recomendaciones}

La percepción sobre la poca pertinencia de la educación universitaria en Honduras en relación a la SAN, está en consonancia con lo que opinan diversos autores sobre la educación superior en Latinoamérica, es una situación preocupante que debería ser tema de discusión en los foros donde convergen los responsables de la educación superior del país.

Las universidades en Honduras tienen en la SAN un espacio para cumplir su función (formación, investigación, extensión). El interés en programas de formación en SAN confirma que hay una demanda no satisfecha que debe ser más estudiada. Mediante un proceso participativo con la sociedad, se debe promover una discusión y proponer las acciones que más convengan. Es necesario definir si es beneficioso y factible diseñar y promover programas de formación en SAN, o es más pertinente que la SAN sea un eje transversal en la educación superior en general.

Es preciso determinar las razones de la poca participación de las universidades en los foros de coordinación sobre la SAN, tal vez desconocen la existencia de los foros o no se ha dimensionado la importancia de su participación y el aporte que pueden ofrecer. También es pertinente profundizar el análisis sobre la generación de información relacionada a la SAN en las universidades; se genera bastante 
información, pero si la misma no está disponible para los tomadores de decisiones o no se publica en los medios adecuados el impacto que genera es mínimo.

Es muy factible y altamente recomendable la inclusión de la formación basada en competencias en los programas universitarios en SAN. Esto podría evolucionar a la implementación de un sistema de certificación de profesionales en SAN bajo el modelo IPMA o cualquier otro modelo. En este proceso podrían desempeñar un rol determinante el gobierno y las ONG, ya que estos son los principales demandantes de profesionales con dicha formación.

Es urgente que el gobierno asuma más responsabilidad en la seguridad alimentaria, ya se cuenta con un marco de políticas favorables, pero es vital mayor coordinación entre las dependencias del estado y entre éstas y el sector privado.

Existe una diversidad de organizaciones que aglutinan a los actores en SAN, pero se requiere más representatividad, especialmente de organizaciones de la sociedad civil. Entre las diversas instancias de coordinación se debe mejorar la comunicación y definir las acciones propicias para generar más impacto a nivel de los territorios e incidir en políticas públicas. Una mayor y mejor coordinación resultará en un mejor aprovechamiento de los recursos disponibles, menor duplicación de esfuerzos y sobretodo generar un mayor impacto en los territorios más desfavorecidos

Cuadro 9. Políticas relacionadas con la Seguridad Alimentaria Nacional (SAN) en Honduras.

Política Principales elementos

Política de Seguridad Alimentaria Nutricional de largo plazo con participación ciudadana (Gobierno de Honduras, 2006).

Política de Estado para el Sector Agroalimentario y el Medio Rural 2004 - 2021 (SAG, 2004?)

Plan Nacional de Desarrollo Agroalimentario 2006-2010

(SAG, 2006)

Política Nacional de Nutrición (Secretaría de salud HN, 2005?a)

Política Nacional de Salud Materno Infantil (Secretaría de salud HN, 2005?b)
Elaborada en 2006 y enmarcada en la estrategia de reducción de la pobreza (ERP). Define las responsabilidades de las Secretarias de Estado y su objetivo es reducir la vulnerabilidad a inseguridad alimentaria en especial de las familias en situación de extrema pobreza.

Su objetivo es reducir la pobreza rural y mejorar la seguridad alimentaria por medio de diferentes acciones: competitividad y calidad del medio rural; fomento productivo e integración de las cadenas agroalimentarias; desarrollo de la agricultura campesina y equidad de género; eficiencia y coordinación público-privada.

Pretende reactivar el sector agroforestal a través del diseño e implementación de políticas que permitan su consolidación, modernización y diversificación productiva.

Política complementaria a la ERP y los objetivos del milenio. Sus ejes transversales son: focalización en mujeres en edad fértil y niños menores de 5 años; equidad de género, intersectorialidad; descentralización y transparencia.

Su objetivo es alcanzar niveles de bienestar físico, mental y social en la población materna e infantil y reducir la morbilidad y mortalidad en esa población. 


\section{Literatura Citada}

AEIPRO (Asociación Española de Ingeniería de Proyectos); IPMA (International Project Management Association). 2006. Bases para la competencia en dirección de proyectos versión 3.0. Traducción y adaptación de D. Jesús Martínez Almela. Editorial UPV. España. 237 p.

ALCSH (Iniciativa América Latina y Caribe sin Hambre). 2009. Foro parlamentario sobre el derecho a la alimentación. Declaración de Santo Domingo, República Dominicana. 10 p.

Álvarez, R. 2004. Formación superior basada en competencias: interdisciplinariedad y trabajo autónomo del estudiante. Revista Iberoamericana de Educación. Consultada el 02/12/2009 en http://www.rieoei.org/deloslectores/648Posada.

Ávila, J., L. Duarte., I. Bonilla., G. Romero y R. Berrios. 2002. Situación de la educación agrícola en Honduras. Ceiba 43(1): 21-25.

Bingxin, Yu., Liangzhi, Y y Shenggen, F. 2010. Toward a typology of food security in developing countries. International Food Policy Research Institute. Discussion paper 00945. $32 \mathrm{p}$.

Bonilla, L. 2003. Sociedad de la información y gestores de información. Revista de Bibliotecología y Ciencias de la Información 4(16): 23-32.

CEPAL. 2009. Anuario estadístico de América Latina y El Caribe.

http://websie.eclac.cl/anuario_estadistico/anuario_2009 /esp/default.asp consultada el 25 de febrero de 2010.

CEPAL y PMA. 2007. El costo del hambre: impacto económico y social de la desnutrición infantil en Centroamérica y República Dominicana. Informe de proyecto. $201 \mathrm{p}$.

Carrión, R. 2003. Prospectiva, pertinencia y calidad de la educación universitaria. Nota científica: Industrial data. 6(1): 103-105.

Correa, E. 2000. El desarrollo rural y la formación de profesionales en América Latina. Pontificia Universidad Javeriana. Seminario Internacional, Bogotá, Colombia. 8 p.

CSUCA (Consejo Superior de Universidades de Centroamérica); INCAP (Instituto de Nutrición de Centroamérica y Panamá); FAO. 2004. Plan de acción universitario (PAU) para la promoción de la seguridad alimentaria y nutricional en Centroamérica 2005-2006. Ciudad de Guatemala. 5 p.

Dijkstra, G. 2007. Evaluación de las Estrategias de Reducción de la Pobreza en América Latina 2006. Informe temático: género y los procesos ERP en Bolivia, Honduras y Nicaragua. Agencia Sueca de Cooperación Internacional. 65 p.
FAO. 1996. Declaración de Roma sobre la seguridad alimentaria y nutricional. Roma Italia. $4 \mathrm{p}$.

FAO. 2004. The state of food insecurity in the world. Monitoring progress towards the world food summit and millennium development goals. Rome, Italy. $40 \mathrm{p}$.

FAO. 2008. Panorama del hambre en América Latina y El Caribe. Iniciativa América Latina y Caribe sin Hambre. Roma, Italia. 63 p.

FAO. $2009_{\mathrm{a}}$. El estado de la inseguridad alimentaria en el mundo 2009. Crisis económicas: repercusiones y enseñanzas extraídas. Roma, Italia. 62 p.

FAO. 2009 . Panorama de la Seguridad Alimentaria y Nutricional en América Latina y el Caribe. Una nueva agenda de políticas públicas para superar la crisis alimentaria. Oficina Regional para América Latina y el Caribe. 73 p.

FAO. 2009. Declaración de la cumbre sobre la seguridad alimentaria. Roma Italia. 8 p.

FAO. 2010. Países de Bajos Ingresos y con Déficit de Alimentos (PBIDA)-Lista para el 2010. http://www.fao.org/countryprofiles/lifdc.asp?lang=es. Sitio web consultado el 25 de febrero de 2010.

FAO y UNESCO. 2003. Education for rural development: towards new policy responses. $462 \mathrm{p}$.

Gobierno de Honduras. 2006. Política de seguridad alimentaria de largo plazo con participación ciudadana. Tegucigalpa, Honduras. $30 \mathrm{p}$.

González, M. 2003. En qué dirección va la seguridad alimentaria. Revista Española de Salud Pública. 77(003): 307-311.

Hoyos, M. 2003. Ciudadanía y participación: más fantasmas para la juventud. Última década: Revista del Centro de Investigación y Difusión Poblacional (19): 1-11.

Izquierdo, M. y A. Márquez. 2000. Indicadores del desarrollo educativo en América Latina y su impacto en los niveles de vida de la población. Revista Electrónica de Investigación Educativa. 2(2): 77-99

Medrano, P. y J. Vallaure. 2006. El coste del hambre. In Trueba, I. (ed.). El fin del hambre en 2025. Un desafío para nuestra generación. Mundi prensa. Madrid, España. p. 145-164.

Oseguera, M. 2005. Diagnóstico sobre políticas de investigación en las universidades. El caso de Honduras. Tegucigalpa, M.D.C. 65 p.

Plata, L. 2003. La pertinencia de la educación superior. Elementos para su comprensión. Revista de la Educación Superior. XXXII-3 (127).

Quiñones, J. y C. V. Alvarez. 2004. Algunas condiciones pedagógicas para la formación y el desarrollo de la investigación en la universidad. Revista Electrónica Actualidades Investigativas en Educación 4(001). 
Ardón, C.R. y Afonso Gallegos, A.: Percepciones sobre programas de formación e institucionalidad en Seguridad Alimentaria

RANSA (Reunión de Alto Nivel sobre Seguridad Alimentaria). 2009. Declaración de Madrid "Seguridad Alimentaria para Todos”. España. 4 p.

República de Honduras. 2006. Estrategia para la reducción de la pobreza; versión actualizada. 57 p.

SAG (Secretaría de Agricultura y Ganadería). 2004?. Política de estado para el sector agroalimentario y el medio rural de Honduras 2004-2021. Tegucigalpa. 189 p.

SAG (Secretaría de Agricultura y Ganadería, HN). 2006. Plan nacional de desarrollo agroalimentario 20062010. Tegucigalpa. $17 \mathrm{p}$.

Sánchez, A. 1993. El proceso de diagnóstico en la elaboración de políticas públicas. Perfiles Latinoamericanos. Facultad Latinoamericana de Ciencias Sociales. (003): 17-36.

Secretaría de Salud, HN. 2005?a. Política nacional de nutrición. Tegucigalpa, Honduras. 25 p.

Secretaría de Salud, HN. 2005?b. Política nacional de salud materno infantil. Tegucigalpa. $25 \mathrm{p}$

Silva, A. 2005. Universidad, democracia y desarrollo. Universidades. Unión de universidades de América Latina (UDUAL). (029): 3-12.
UNAT-SAN (Unidad Técnica de Seguridad Alimentaria y Nutricional). 2009. Plan de trabajo para el desarrollo de actividades en el marco de la implementación de la política de seguridad alimentaria y nutricional. Tegucigalpa, Honduras. 14 p.

UNESCO. 1996. Declaración de conferencia regional sobre políticas y estrategias de la educación superior en América Latina y El Caribe. Habana, Cuba.

UNESCO. 2008. Declaración de conferencia regional de la educación superior en América Latina y El Caribe. Cartagena, Colombia. 10 p.

UN Millennium Project. 2005. Halving Hunger: It Can Be Done. Summary version of the report of the Task Force on Hunger. The Earth Institute at Columbia University, New York, USA. 32 p.

Yániz, C. 2008. Las competencias en el currículo universitario: implicaciones para diseñar el aprendizaje y para la formación del profesorado. Revista de Docencia Universitaria. Número monográfico $1^{\circ}$. Consultado el $02 / 12 / 2009$ en http://www.redu.m.es/Red_U/m.

Recibido para publicación el 15 de marzo de 2010 